\title{
Ensino de Programação Front-end através de jogos digitais: Um relato de experiência na Escola de Programação do LAIS/HUOL
}

\author{
Fernando Lucas de Oliveira Farias ${ }^{1}$, Danieli Silva de Souza Rabelo², Ricardo Alexsandro \\ de Medeiros Valentim² e Isabel Dillmann Nunes ${ }^{1}$
}

Instituto Metrópole Digital - Universidade Federal do Rio Grande do Norte (UFRN) Av. Sen. Salgado Filho, 3000 - Lagoa Nova, CEP: 59.078-970 - Natal - RN - Brasil ${ }^{1}$

Laboratório de Inovação Tecnológica em Saúde (LAIS) - Universidade Federal do Rio Grande do Norte (UFRN)

Av. Nilo Peçanha, 620 - Petrópolis, CEP: 59.012-300 - Natal - RN - Brasil ${ }^{2}$

\{fernandoo.mcp, rabrlodanni, ricardo. lahb, beldillnunes\} @gmail.com

\begin{abstract}
The use of digital games can offer students new ways of learning, perceiving, reflecting and creating meaning in the active construction of their knowledge. In this sense, the present work deals with the teaching of front-end programming for students graduated from the most diverse areas of knowledge, organized in multidisciplinary teams supported by games. The methodology of the experiment is guided by Action Research, Bloom taxonomy and reaction evaluation performed at the end of each educational scenario, involving the use of the following digital games: CSS Diner, Flexbox Defense and Flexbox Froggy. The results indicate that games are capable of promoting a more meaningful, profitable and playful learning in the construction of knowledge, skills and attitudes.
\end{abstract}

Resumo. A utilização de jogos digitais pode proporcionar aos estudantes novas formas de aprender, perceber, refletir e criar significado na construção ativa do seu conhecimento. Neste sentido, o presente trabalho trata do ensino de programação frontend para estudantes egressos das mais diversas áreas do conhecimento, organizados em equipes multidisciplinares apoiado por games. A metodologia do experimento é norteada pela Pesquisa-ação, taxonomia de Bloom e avaliação de reação realizada ao final de cada cenário educacional, envolvendo a utilização dos seguintes jogos digitais: CSS Diner, Flexbox Defense e o Flexbox Froggy. Os resultados indicam que os jogos são capazes de promover uma aprendizagem mais significativa, proficua e lúdica na construção do conhecimento, habilidades e atitudes.

\section{Introdução}

O aprendizado de programação tem se demonstrado uma habilidade valiosa à formação plena de qualquer profissional, não se restringindo à área de computação; principalmente por se tratar de uma habilidade transversal relacionada à capacidade de sistematizar, analisar e resolver problemas em diferentes níveis de abstração e granularidade [Raabe et al. 2017]

O desenvolvimento de práticas educativas que procurem estabelecer o pleno fluxo da experiência nos processos de ensino e aprendizagem tem sido um grande desafio; principalmente pelos diferentes níveis de maturidade e pensamento computacional que estudantes apresentam durante a fase de imersão inicial, seja no estudo da lógica ou linguagem de programação. 
Neste contexto, a utilização de jogos digitais pode proporcionar aos estudantes novas formas de aprender, perceber, refletir e criar significado na construção ativa do seu conhecimento, consistindo numa estratégia capaz de promover uma aprendizagem mais rápida e próxima da vida real dos aprendizes [Morán 2013].

McGonigal (2012), relata que a humanidade passa cerca de três bilhões de horas semanais jogando. Tal indicador qualifica e reforça o papel relevante que os jogos desempenham na sociedade atual, seja na perspectiva do entretenimento ou seu potencial educacional.

Não obstante, o presente trabalho tem como objetivo principal apresentar um relato de experiência no ensino de programação front-end ${ }^{1}$ através de jogos digitais para estudantes provenientes das mais diversas áreas do conhecimento que, atuam como pesquisadores em equipes multidisciplinares no desenvolvimento de sistemas web para área de saúde no Laboratório de Inovação Tecnológica em Saúde - LAIS sediado no Hospital Universitário Onofre Lopes - HUOL da Universidade Federal do Rio Grande do Norte - UFRN.

Os jogos digitais selecionados foram: o CSS Diner ${ }^{2}$ que trabalha conteúdos relacionados a seletores $\mathrm{CSS}^{3}$; o Flexbox Froggy ${ }^{4}$ que auxilia no aprendizado de regras CSS com Flexible Box Layout Model - Flexbox e o Flexbox Defense ${ }^{5}$ que trabalha o conteúdo CSS Flexbox numa perspectiva interdisciplinar através da eliminação de inimigos posicionando torres de defesa com regras CSS, exigindo que o estudante estabeleça estratégias de guerra para avançar nas fases e/ou desafios.

Os resultados do experimento apontam uma percepção positiva acerca do design, jogabilidade, mecânicas e aspectos pedagógicos dos jogos "CSS Diner, Flexbox Defense e Flexbox Froggy"; sendo capazes de criar um fluxo de experiência que manteve os aprendizes participantes do experimento engajados nos processos de ensino e aprendizagem.

O trabalho traz como contribuição principal, uma análise empírica da efetividade dos jogos digitais selecionados na aquisição de conhecimentos em programação front-end; sendo que não foram identificados estudos brasileiros que apresentem cenários educacionais envolvendo a avaliação do potencial educacional dos jogos digitais no ensino de programação para web objeto de nossa investigação, sendo a grande maioria voltada para o ensino do pensamento computacional, lógica e programação para iniciantes.

\section{Referencial Teórico}

A construção de cenários educacionais na perspectiva da aprendizagem baseada em desafios, problemas reais e/ou jogos em aulas roteirizadas, apoiadas por uma metodologia ativa de ensino, a exemplo da sala de aula invertida são primordiais à promoção de maior engajamento, pensamento crítico e postura reflexiva dos aprendizes [Moran 2013].

\footnotetext{
${ }^{1}$ Paiva (2018), cita em seu estudo que “o front-end developer foca-se no desenvolvimento das interfaces gráficas com as quais o utilizador opera.

2 Disponível em: <https://flukeout.github.io/> Acesso em: 12 dez. 2018

${ }^{3}$ Silva (2018), define CSS ou regra de estilo ou de estilização, como sendo a unidade básica de uma folha de estilo. Sendo a menor porção de código capaz de produzir efeito de estilização na página.

${ }^{4}$ Disponível em: <https://flexboxfroggy.com/> Acesso em: 12 dez. 2018

${ }^{5}$ Disponível em: <http://www.flexboxdefense.com/> Acesso em: 12 dez. 2018
} 
Uzunca e Jansen (2016 apud Silva; Fernandes e Santos, 2018) esclarecem que os jogos são uma nova forma de aprender, uma vez que proporcionam: (i) o aprendizado em uma realidade virtual ou papel simulado, criando uma experiência profícua para o jogador, pois, ele pode sempre recomeçar; (ii) "aprender fazendo", envolvendo-se, motivando-se e entretendo-se; (iii) desenvolver a capacidade de encontrar e utilizar a informação sem a necessidade de memorização num viés menos instrucionista; e (iv) simular ambientes complexos/caros e situações perigosas/críticas.

Neste contexto, o jogo digital CSS Diner foi selecionado especialmente por contextualizar o uso de seletores CSS, pseudoclasses e pseudoelementos por meio de desafios onde o jogador, tendo conhecimento prévio em tais tecnologias é convidado a estruturar comandos apropriados na solução dos problemas propostos no sequenciamento didático, gerando animações e dicas que tornam o processo lúdico e centrado na experiência do aprendiz.

De Paula e Valente (2015) relatam que os jogos digitais podem contribuir com a nova perspectiva da Educação, sendo capazes de colocar os estudantes no centro da ação, protagonistas na construção ativa do seu conhecimento.

A aprendizagem ativa baseada em jogos digitais utilizada como estratégia metodológica na elaboração dos cenários educacionais descritos na tabela 1, teve papel primordial ao protagonismo do estudante na construção de seu conhecimento, desenvolvimento de habilidades relacionadas à programação para web, conectando conteúdos vistos em desafios anteriores, mantendo-o engajado e atento às orientações relacionadas à solução do problema proposto, enquanto constrói o comando necessário para avançar na fase seguinte.

Connoly et al. (2012) em seu levantamento de trabalhos sobre os impactos dos jogos digitais na Educação, perceberam um número significativo de trabalhos apontando evidências empíricas da efetividade dos jogos digitais na aquisição de conhecimentos específicos.

Nesta perspectiva, os cenários educacionais interdisciplinares foram desenvolvidos para ensino de conteúdos específicos conexos à programação front-end, levando em consideração o conhecimento prévio dos participantes em lógica e programação para iniciantes, potencial pedagógico dos jogos digitais selecionados frente aos objetivos de aprendizagem traçados e ausência de conhecimento anterior em programação para web.

\subsection{Teoria do Fluxo da Experiência "Estado de Fluxo"}

A teoria do fluxo da experiência contribuiu bastante para o desenho de cenários educacionais que procuram manter o aluno motivado e engajado nos processos de ensino e aprendizagem por intermédio de experiências significativas, profícuas e lúdicas que procuram estimular o desenvolvimento de competências, habilidades e atitudes no aprendiz.

Lumsden (1994), relata que a motivação está associada com a vontade de envolver-se com o processo de aprendizagem, isto é, os motivos ou objetivos que apoiam o envolvimento ou não em atividades acadêmicas.

Csikszentimihalyi (1990) esclarece que se o desafio do jogo estiver aquém das habilidades do jogador, ele ficará entediado, porém, se estiver além, ele ficará frustrado e desistirá do que lhe foi proposto. 
VIII Congresso Brasileiro de Informática na Educação (CBIE 2019)

Anais do XXV Workshop de Informática na Escola (WIE 2019)

\title{
3. Metodologia
}

A metodologia utilizada no desenvolvimento do experimento foi norteada pela pesquisa-ação. Delimitando-se o problema de pesquisa à seleção de jogos digitais que apoiem o ensino de programação front-end para pesquisadores de cursos não relacionados a computação e a avaliação do design, jogabilidade, mecânicas e aspectos pedagógicos dos jogos utilizados através de um survey ${ }^{6}$ disponibilizado ao final de cada cenário educacional na avaliação de reação.

A pesquisa-ação traz inúmeras contribuições na perspectiva da pesquisa aplicada em diferentes área de atuação, inclusive para Educação onde

\begin{abstract}
“os pesquisadores em educação estariam em condição de produzir informações e conhecimentos de uso mais efetivo, inclusive ao nível pedagógico. Tal orientação contribuiria para o esclarecimento das microssituações escolares e para a definição de objetivos de ação pedagógica e de transformações mais abrangentes" (THIOLLENT, 1986, p.75)
\end{abstract}

Os cenários educacionais com jogos digitais descritos na Tabela 1 foram planejados baseados na taxonomia dos objetivos educacionais proposta por Bloom et al. (1956) contribuindo no planejamento, organização e controle dos objetivos de aprendizagem, sendo estruturada em três domínios: Cognitivo, Afetivo e Psicomotor; em nosso estudo enfatizamos análise dos aspectos relacionados ao domínio cognitivo que concentra sua análise na relação do aprender e dominar um conhecimento específico pelo estudante.

Tabela 1: Planejamento dos Cenários Educacionais com Jogos Digitais

\begin{tabular}{|c|c|c|c|c|}
\hline $\begin{array}{c}\text { Cenário } \\
\text { Educacional } \\
\text { / Horas aula }\end{array}$ & $\begin{array}{c}\text { Jogo } \\
\text { Digital }\end{array}$ & $\begin{array}{c}\text { Objetivos de } \\
\text { Aprendizagem }\end{array}$ & Conteúdos desenvolvidos pelo jogo & $\begin{array}{l}\text { Critérios de } \\
\text { avaliação }\end{array}$ \\
\hline $\begin{array}{l}\text { Trabalhando } \\
\text { com seletores } \\
\text { CSS, } \\
\text { Pseuclasses e } \\
\text { Pseudoeleme } \\
\text { ntos / } 6 \mathrm{~h} \\
\text { (aula) e } 2 \mathrm{~h} \\
\text { (jogo) }\end{array}$ & $\begin{array}{l}\text { CSS } \\
\text { Diner }\end{array}$ & $\begin{array}{l}\text { - Criar seletores CSS } \\
\text { para elementos; } \\
\text { - Criar seletores CSS } \\
\text { para \#ID; } \\
\text { - Criar seletores CSS } \\
\text { para .class; } \\
\text { - Criar seletores CSS * } \\
\text { universal; } \\
\text { - Criar seletores CSS } \\
\text { para hierarquia } \\
\text { ancestral descendente; } \\
\text { irmão irmão; irmão + } \\
\text { irmão seguinte; pai > } \\
\text { filho. } \\
\text { - Criar seletores CSS } \\
\text { para pseudoclasses; }\end{array}$ & 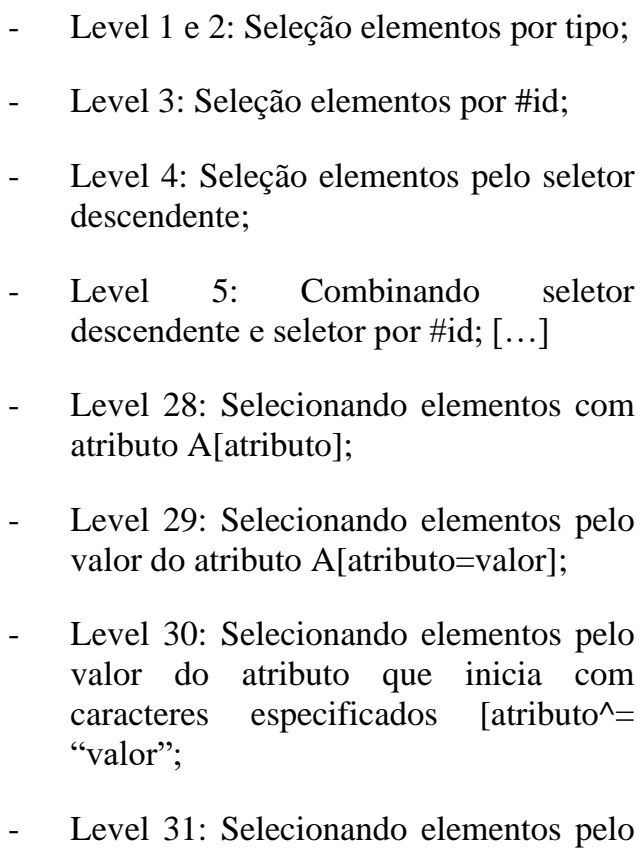 & $\begin{array}{lr}\text { - Avaliação } & \text { processual de } \\
\text { forma contínua, } & \text { naseada ra } \\
\text { participação do } & \text { aprendiz } \\
\text { evolução nas } & \text { missões e levels } \\
\text { no CSS Diner; } \\
\text { - Conclusão dos } \\
\text { desafios da } \\
\text { Missão 1 (Level } \\
\text { 1 a Level 14); } \\
\text { - Conclusão da } \\
\text { Missão 2 (Level } \\
\text { 15 a 24); }\end{array}$ \\
\hline
\end{tabular}

\footnotetext{
${ }^{6}$ Disponível em: <http://bit.ly/ppgite-artigo-lais-jogos> Acesso em: 14 dez. 2018
} 
VIII Congresso Brasileiro de Informática na Educação (CBIE 2019)

Anais do XXV Workshop de Informática na Escola (WIE 2019)

\begin{tabular}{|c|c|c|c|c|}
\hline & & & $\begin{array}{l}\text { valor do atributo que termina com } \\
\text { caracteres especificados [atributo } \$= \\
\text { "valor"; } \\
\text { - Level 32:Selecionando elementos pelo } \\
\text { valor do atributo que contém os } \\
\text { caracteres especificados [atributo*= } \\
\text { "valor"; }\end{array}$ & $\begin{array}{l}\text { Conclusão da } \\
\text { Missão } 3 \text { (Level } \\
25 \text { a 32); }\end{array}$ \\
\hline $\begin{array}{l}\text { Trabalhando } \\
\text { a construção } \\
\text { de seletores } \\
\text { CSS Flexbox } \\
\text { básico / } 3 \mathrm{~h} \\
\text { (aula) e } 1 \mathrm{~h} \\
\text { (jogo) }\end{array}$ & $\begin{array}{c}\text { Flexbox } \\
\text { Froggy }\end{array}$ & $\begin{array}{l}\text { - Criar seletores CSS } \\
\text { Flexbox com flex- } \\
\text { direction; } \\
\text { - Criar seletores CSS } \\
\text { Flexbox com flex } \\
\text { order; } \\
\text { - Criar seletores CSS } \\
\text { Flexbox com flex- } \\
\text { wrap; } \\
\text { Criar seletores CSS } \\
\text { Flexbox com flex- } \\
\text { grow; } \\
\text { Criar seletores CSS } \\
\text { Flexbox com flex- } \\
\text { shrink; } \\
\text { Criar seletores CSS } \\
\text { Flexbox com } \\
\text { justify-content; } \\
\text { Criar seletores CSS } \\
\text { Flexbox com align- } \\
\text { self; }\end{array}$ & $\begin{array}{l}\text { - Level } 1 \text { a 4: Criando regra CSS Flexbox } \\
\text { com Justify-content; } \\
\text { - Level 5: Criando regra CSS Flexbox com } \\
\text { align-items; } \\
\text { - Level } 6 \text { e 7: Criando regra CSS Flexbox } \\
\text { com justify-content e align-items; [...] } \\
\text { - Level 22: Criando regra CSS Flexbox com } \\
\text { align-content; } \\
\text { - Level 23: Criando regra CSS Flexbox com } \\
\text { flex-direction e align-content; } \\
\text { - Level 24: Criando regra CSS Flexbox com } \\
\text { justify-content, align-items, flex-direction, } \\
\text { order, align-self, flex-wrap, flex-flow e align- } \\
\text { content. }\end{array}$ & $\begin{array}{l}\text { - Avaliação } \\
\text { processual de } \\
\text { forma contínua, } \\
\text { baseada na } \\
\text { participação do } \\
\text { aprendiz e } \\
\text { evolução nas } \\
\text { missões e levels } \\
\text { do Flexbox } \\
\text { Froggy; }\end{array}$ \\
\hline $\begin{array}{l}\text { Trabalhando } \\
\text { a construção } \\
\text { de seletores } \\
\text { CSS Flexbox } \\
\text { avançados / } \\
\text { 2h (aula) e } \\
\text { 50min (jogo) }\end{array}$ & $\begin{array}{l}\text { Flexbox } \\
\text { Defense }\end{array}$ & 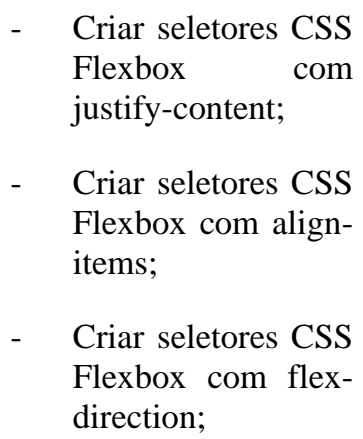 & $\begin{array}{l}\text { - Level } 1 \text { a 3: Criando regras CSS Flexbox } \\
\text { para movimentar as torres de guerra } \\
\text { utilizando justify-content; } \\
\text { - Level 4: Criando regras CSS Flexbox para } \\
\text { movimentar as torres de guerra utilizando } \\
\text { align-items; } \\
\text { - Level 5: Criando regras CSS Flexbox para } \\
\text { movimentar as torres de guerra utilizando } \\
\text { justify-content e align-items; [...] }\end{array}$ & $\begin{array}{l}\text { - Avaliação } \\
\text { processual de } \\
\text { forma contínua, } \\
\text { baseada na } \\
\text { participação do } \\
\text { aprendiz e } \\
\text { evolução nas } \\
\text { missões e levels } \\
\text { do Flexbox } \\
\text { Defense; }\end{array}$ \\
\hline
\end{tabular}

Fonte: Elaborado pelos autores

Os jogos digitais “CSS Diner, Flexbox Froggy e Flexbox Defense”, ilustrados na Figura 2, foram selecionados levando em consideração seus aspectos pedagógicos e educacionais, possibilidade de trilhas de aprendizagem personalizadas para cada estudante, ensino adaptativo 
dos conteúdos propostos, boas mecânicas e jogabilidade que fossem capazes de proporcionar uma experiência lúdica e interativa aos aprendizes.

Figura 2: Jogos Digitais para Ensino de Programação Front-end
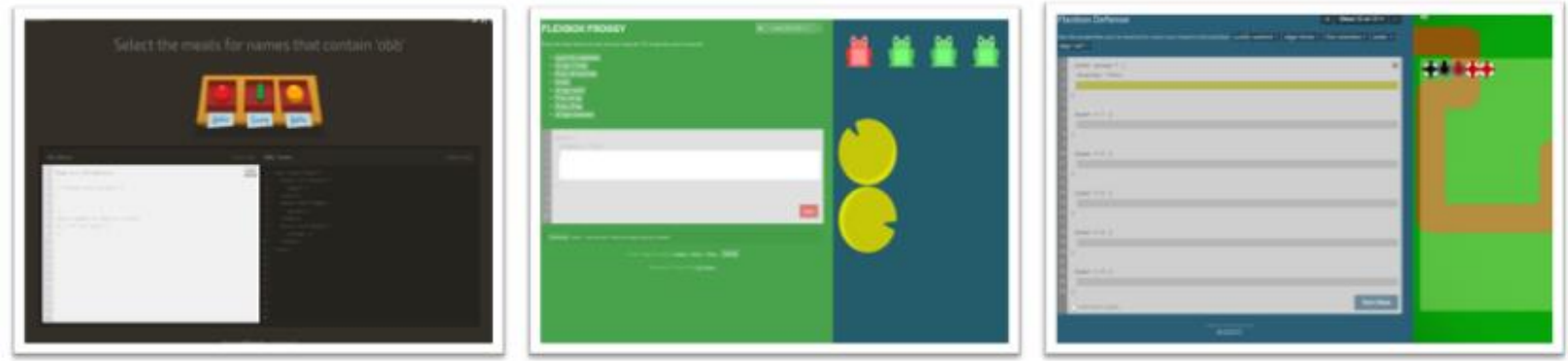

Fonte: Elaborado pelos autores

No encerramento de cada cenário educacional, o jogo foi avaliado por cada aprendiz participante da intervenção por meio de uma avaliação de reação que teve como objetivo principal colher informações acerca de sua percepção e experiência na utilização de jogos digitais como estratégia à consolidação de competências relacionadas ao aprendizado ativo de tecnologias para desenvolvimento web front-end (CSS, Flexbox,...) estimulada pelos jogos: CSS Diner, Flexbox Froggy e Flexbox Defense.

O instrumento para avaliação da experiência dos participantes foi estruturado nas seguintes dimensões: pedagógica, design, mecânicas, jogabilidade e seus resultados são discutidos na seção de análise e discussão dos dados deste trabalho.

\section{Análise e Discussão dos Dados}

Em nosso experimento foi possível identificar, após análise dos dados da avaliação de reação e observação do comportamento dos estudantes nas práticas educativas desenvolvidas, o papel singular dos jogos selecionados, enquanto impulsionadores do pleno fluxo da experiência, mantendo-os engajados durante todo cenário educacional, conforme relatos e dados coletados.

Os jogos digitais foram disponibilizados para estudantes egressos das mais diversas áreas do conhecimento, sendo 33,3\% da Engenharia Biomédica e Mecânica, 16,7\% Tecnologia da Informação e Ciência e Tecnologia; reforçando o potencial inovador da proposta pedagógica que consiste no ensino de programação front-end para estudantes não pertencentes a cursos de computação, ver Gráfico 1.
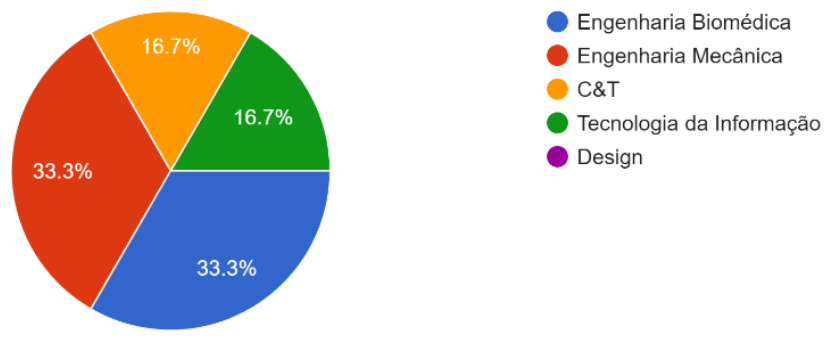

Gráfico 1: Perfil do Jogador - Cursos frequentados pelos participantes do experimento 
VIII Congresso Brasileiro de Informática na Educação (CBIE 2019)

Anais do XXV Workshop de Informática na Escola (WIE 2019)

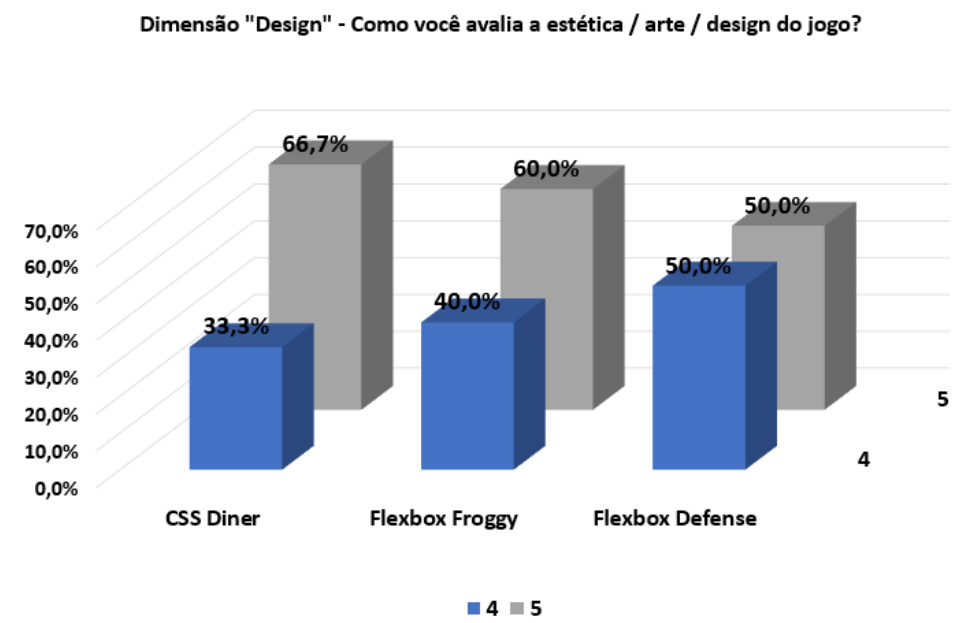

Gráfico 2: Dimensão "Design" - Avaliação da estética / arte / design dos jogos digitais

O Gráfico 2 indica uma aceitação maior numa escala de 1 - Não me agradou muito a 5 Gostei bastante para o jogo CSS Diner que teve 66,6\% de aceitação máxima em detrimento ao Flexbox Defense que teve a menor aceitação. A variação de percepção pode estar relacionada ao tempo de manutenção evolutiva / desenvolvimento das plataformas; uma vez que o CSS Diner é mantido há cinco anos, Flexbox Froggy e o Flexbox Defense há três anos pelos desenvolvedores.

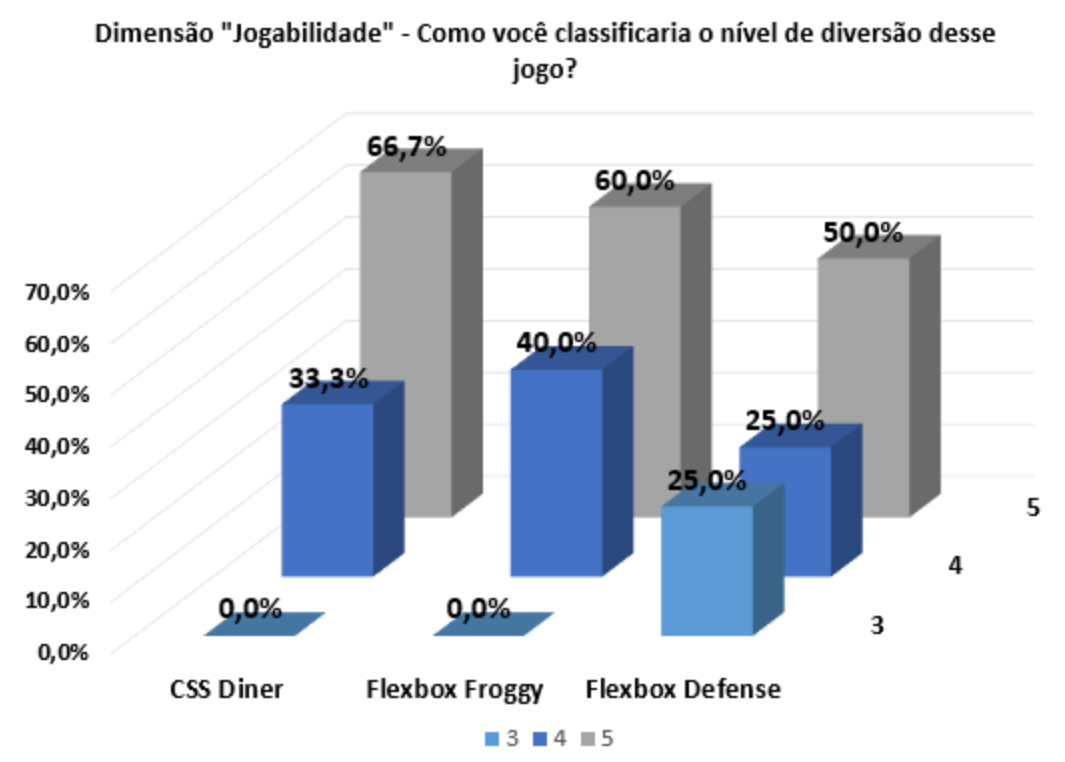

Gráfico 3: Dimensão "Jogabilidade” - Avaliação do nível de diversão dos jogos

O Gráfico 3 reforça os aspectos positivos avaliados em nossos cenários educacionais, conforme Tabela 2, à medida em que os jogos digitais avaliados são capazes de promover a manutenção do fluxo da experiência, tornando os estudantes mais atentos aos objetivos de aprendizagem e centrados na construção de suas habilidades, competências e atitudes propostas no aprendizado de programação front-end, ver as Figuras 3 e 4. 
Neste sentido, identificamos que o Jogo Flexbox Defense é considerado o menos divertido pelos participantes numa escala de 1 - Pouco Divertido a 5 - Muito Divertido, fato associado ao baixo número de desafios existentes, apenas doze; considerando que o CSS Diner possui trinta e dois níveis, o Flexbox Froggy apresenta vinte e quatro níveis, resultando em uma experiência com menor densidade, criando trilhas de aprendizagem curtas, comprometendo a possibilidade de promover um ensino adaptativo e personalizado mais amplo.

Tabela 2: Aspectos positivos e negativos dos jogos digitais na percepção dos participantes

\begin{tabular}{|c|c|c|}
\hline $\begin{array}{c}\text { Jogo } \\
\text { Digital }\end{array}$ & Aspectos Positivos & Aspectos Negativos \\
\hline \multirow{3}{*}{$\begin{array}{l}\text { CSS } \\
\text { Diner }\end{array}$} & $\begin{array}{l}\text { "Ele aborda com boa profundidade os } \\
\text { seletores css e é uma boa prática para } \\
\text { quem está iniciando" }\end{array}$ & $\begin{array}{l}\text { "Em algumas questões fiquei presa por muito tempo por } \\
\text { estar errando algo mínimo. O jogo poderia dar uma dica } \\
\text { nesses casos" }\end{array}$ \\
\hline & $\begin{array}{l}\text { "Jogo bem completo com vários níveis } \\
\text { de complexidade" }\end{array}$ & "Alguns desafios não eram muito claros" \\
\hline & $\begin{array}{l}\text { "De forma geral é ótimo para reforçar } \\
\text { os conhecimentos adquiridos, é prático } \\
\text { e intuitivo." }\end{array}$ & $\begin{array}{l}\text { "Em algumas situações o jogo exige um caractere } \\
\text { exatamente igual ao o do gabarito e tive que buscar no } \\
\text { google. Por exemplo, a resposta continha o caractere '^ ', } \\
\text { porém o computador que eu usei o inseria de forma menor } \\
\text { do que o do gabarito e o jogo não aceitava, mesmo sendo } \\
\text { o mesmo caractere, então tive que ir no Google, pesquisar } \\
\text { um maior, copiar e colar." }\end{array}$ \\
\hline \multirow[b]{3}{*}{$\begin{array}{l}\text { Flexbox } \\
\text { Froggy }\end{array}$} & $\begin{array}{l}\text { "A última fase é a melhor aplicar todo } \\
\text { o conhecimento sobre o assunto para } \\
\text { finalizá-lo." }\end{array}$ & "Não possuir as dicas em todas as fases." \\
\hline & $\begin{array}{l}\text { "Visual agradável e fácil compreensão } \\
\text { na jogabilidade" }\end{array}$ & "Não ter uma versão em português." \\
\hline & $\begin{array}{l}\text { "Fazer versões em outros idiomas, } \\
\text { sobretudo o português. O jogo facilita } \\
\text { bastante a imersão dos conteúdos, } \\
\text { fazendo com que tire um pouco a } \\
\text { necessidade de ficar lendo } \\
\text { documentação da linguagem e afins, } \\
\text { ou seja, faz com que o jogador aprenda } \\
\text { sem perceber e de forma divertida." }\end{array}$ & - \\
\hline $\begin{array}{l}\text { Flexbox } \\
\text { Defense }\end{array}$ & $\begin{array}{l}\text { Estratégia, associado com o } \\
\text { conhecimento em flexbox. }\end{array}$ & $\begin{array}{l}\text { O jogo poderia ter a opção de aumentar a velocidade com } \\
\text { que os tanques andam... para não perder tanto tempo. }\end{array}$ \\
\hline
\end{tabular}

Fonte: Elaborado pelos autores 


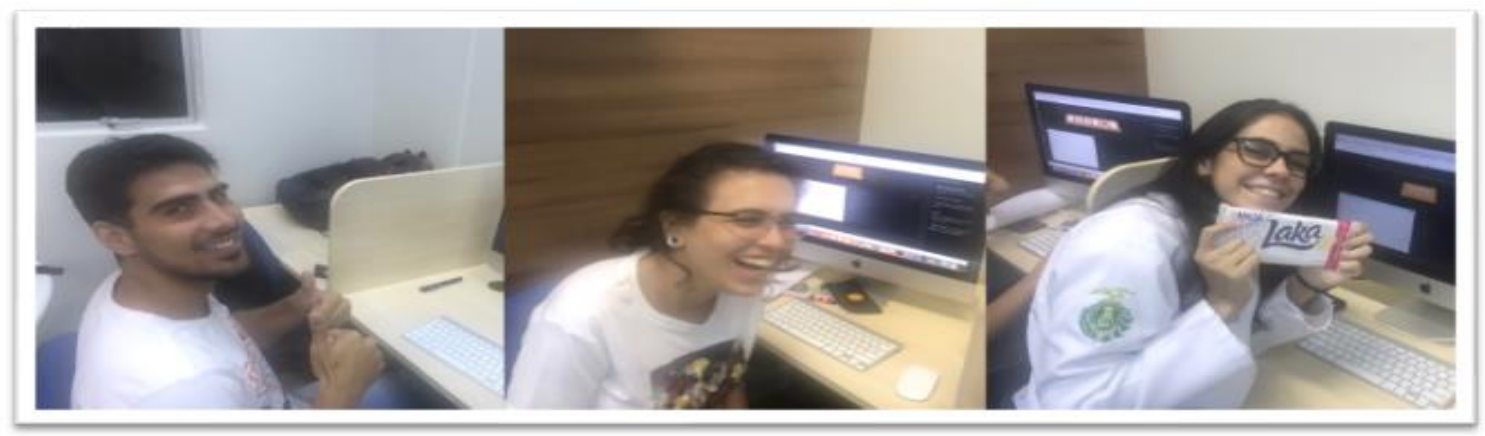

Figura 3: Pesquisadores participantes após cenário Educacional com CSS Diner
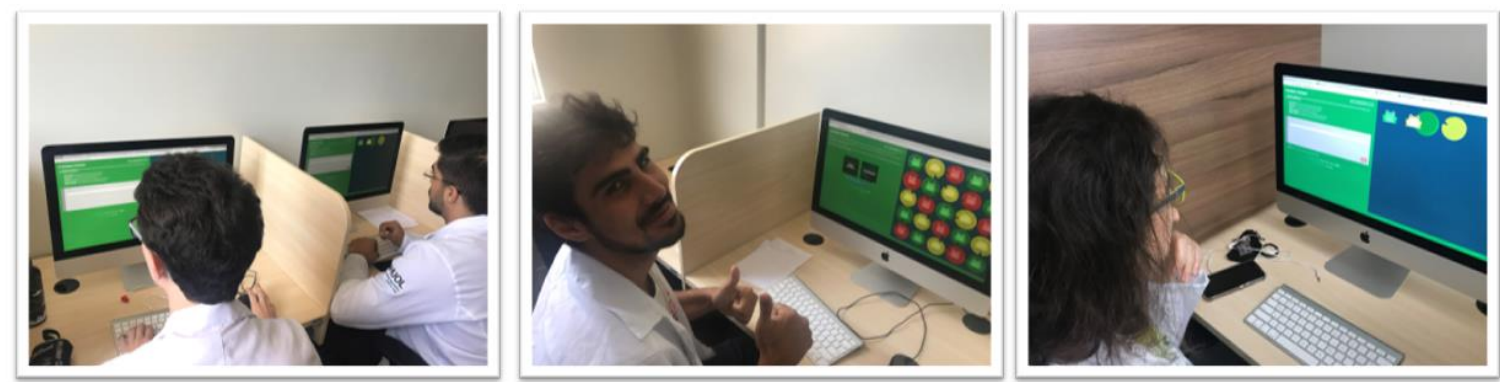

Figura 4: Pesquisadores durante cenário Educacional com CSS Flexbox Froggy

Identificamos uma percepção positiva por parte dos aprendizes no que tange às mecânicas dos jogos educacionais utilizados, principalmente no que diz respeito à estruturação das fases, sistema de recompensas, habilidades trabalhadas durante o uso do game. O jogo com maior aceitação de suas mecânicas foi o CSS Diner, enquanto o Flexbox Defense teve uma menor aceitação, motivada principalmente pelo menor tempo de desenvolvimento, manutenção evolutiva e número de voluntários envolvidos, conforme discutido na análise do Gráfico 2.

\section{Considerações finais}

Os resultados do experimento indicam uma percepção positiva acerca do design, jogabilidade e mecânicas dos jogos "CSS Diner, Flexbox Defense e Flexbox Froggy" utilizados em nossos cenários educacionais planejados para ensino de programação front-end, em especial seletores básicos, avançados, complexos CSS e o CSS Flexbox.

Não obstante, proporcionaram uma aprendizagem adaptativa e criação de um fluxo de experiência que manteve os aprendizes engajados nos processos de ensino e aprendizagem, sendo a principal contribuição deste trabalho apresentar e avaliar jogos digitais que apoiem práticas educativas digitais no ensino de programação front-end, seja para alunos dos cursos de computação ou estudantes das mais diversas áreas do conhecimento.

Em trabalhos futuros, os cenários educacionais desenvolvidos serão aprimorados e calibrados para novas situações de aprendizagem, levando em consideração às lições apreendidas e feedbacks coletados; bem como avaliar novos jogos com potencial educacional no contexto do ensino de programação front-end e criação de um repositório colaborativo para centralização e compartilhamento dos cenários educacionais desenvolvidos e lições aprendidas registradas pelos pesquisadores tutores da escola de programação do LAIS/HUOL. 
VIII Congresso Brasileiro de Informática na Educação (CBIE 2019)

Anais do XXV Workshop de Informática na Escola (WIE 2019)

\section{Referências}

Bloom, B.S.et al (1956). Taxonomy of educational objectives: The classification of educational goals. Handbook 1: Cognitive domain. New York: David McKay.

Connolly, Thomas M. et al (2012). A systematic literature review of empirical evidence on computer games and serious games. Computers \& Education, v. 59, n. 2, p. 661-686.

Czisikszentmihalyi, M (1990). Flow-The Psychology of Optimal Experience.

Csikszentmihalyi, Mihaly (2014). Toward a psychology of optimal experience. In: Flow and the foundations of positive psychology. Springer, Dordrecht, p. 209-226.

Da Silva, Carlos Antonio Fernandes; Da Silva, Leandro Dias; Martins, João Carlos Diniz (2018). Aplicação do The Huxley no ensino de programação para alunos do curso técnico em informática para internet. In: Proceedings of SBGames 2018.

De Paula, Bruno Henrique; Valente, José Armando (2016). Jogos digitais e educação: uma possibilidade de mudança da abordagem pedagógica no ensino formal. Revista Iberoamericana de Educação, v. 70, n. 1, p. 9-28.

Lumsden, Linda S (1994). Student Motivation To Learn. Eric Digest, Number 92. 1994.

Mcgonigal, Jane (2012). A realidade em jogo: por que os games nos tornam melhores e como eles podem mudar o mundo. Rio de Janeiro: Bestseller.

Morán, J (2013). Metodologias ativas para uma aprendizagem mais profunda. Disponível em: $<$ http://bit.ly/2MkHKxG>. Acesso em: 07 dez. 2018.

Paiva, Moisés Silva de (2018). Técnicas e tecnologias para desenvolvimento de Front-End na empresa PontoPR-Inovação Digital. Dissertação de Mestrado.

Raabe, A.L. et al. (2017). Referenciais de Formação em Computação: Educação Básica. In: Sociedade Brasileira de Computação.

Silva, Rodrigo Ribeiro; Fernandes, Juliana; Santos, Rodrigo (2018). Panorama da Utilização de Jogos Digitais no Ensino de Programação no Nível Superior na Última Década: Uma Revisão Sistemática da Literatura. In: Brazilian Symposium on Computers in Education (Simpósio Brasileiro de Informática na Educação-SBIE), p.535.

Silva, Maurício Samy (2018). Fundamentos de HTML5 e CSS3. Novatec Editora.

Thiollent, M (1986). Metodologia da pesquisa-ação”. São Paulo: Cortez Editora.

Uzunca, B.; Jansen, S (2016). How do Ecosystem Dynamics work in Serious Gaming Ecosystems? Challenges and Opportunities. Strategic Management Society. 